\title{
Effect of photoperiod on neural estrogen and progestin receptor immunoreactivity in female Syrian hamsters
}

\author{
Robert A. Mangels, J. Bradley Powers *, Jeffrey D. Blaustein \\ Department of Psychology, Center for Neuroendocrine Studies, and Neuroscience and Behavior Program, University of Massachusetts, Box 37710, \\ Amherst, MA 01003-7710, USA
}

Accepted 17 March 1998

\begin{abstract}
This study explored the possibility that reduced behavioral responsiveness to estradiol and progesterone in female Syrian hamsters exposed to a short photoperiod is associated with a reduction in the concentration of neural steroid receptors. The effects of long and short photoperiod (LP; SP) exposure on steroid receptor immunoreactivity were examined in the ventromedial hypothalamus (VMH), medial tuberal region $(\mathrm{mTu})$, medial preoptic area (mPOA), medial nucleus of the amygdala (mAMYG), and the arcuate nucleus (ARC) of ovariectomized hamsters. In Experiment 1, exposure to SP for ten weeks attenuated the lordosis response following sequential treatment with estradiol and progesterone. In a separate group of animals not given hormones, SP decreased the staining intensity of estrogen receptor immunoreactive (ERIR) cells in the mPOA while increasing the number of detectable ERIR cells in part of the mAMYG. In Experiment 2, SP diminished the lordosis response as it did in Experiment 1. One week later, the same females were administered estradiol systemically to induce progestin receptors (PR). Animals housed in SP showed significantly reduced progestin receptor immunoreactivity (PRIR) in the VMH, mTu, mPOA, mAMYG, and ARC. Experiment 3 examined whether the results of Experiment 2 might have been influenced by photoperiodic effects on peripheral metabolism of estradiol. Among hamsters housed in LP or SP, PRs were induced by estradiol implanted unilaterally in the medial basal hypothalamus, thus bypassing possible photoperiodic effects on peripheral estradiol availability. This treatment resulted in significantly fewer cells with detectable PRIR in the VMH and mPOA of SP females, suggesting that the photoperiodic influences on PR induction observed in Experiment 2 do not depend on alterations in the peripheral availability of estradiol. (C) 1998 Elsevier Science B.V. All rights reserved.
\end{abstract}

Keywords: Lordosis response; Photoperiod; Neural estrogen receptor; Neural progestin receptor; Syrian hamster; Medial preoptic area; Ventromedial hypothalamus

\section{Introduction}

Syrian hamsters are seasonal breeders, reproducing only at certain times of the year. Seasonal breeders time their bouts of sexual activity with respect to their species' gestational length to give birth during the spring and summer when food resources are high and the demands for energy to fuel homeostatic processes, such as thermoregulation, are low. This increases the number of calories available to support lactation [11,12]. Additionally, many species exhibit other seasonal alterations in metabolism, activity, and body mass $[4,11,36]$. These adaptations maximize efficient use of available energy and increase the chances of survival.

\footnotetext{
* Corresponding author. Fax: +1-413-545-0996; E-mail: jbp@psych.umass.edu
}

When exposed for six or more weeks to short or winter-like photoperiods, or nightly melatonin signals of appropriate duration, male and female Syrian hamsters experience a decrease in gonadotropin secretion which ultimately results in complete reproductive collapse [34,46]. With decreased gonadotrophic stimulation, gonadal atrophy occurs, gametogenesis is arrested, and the secretion of steroid hormones is sharply diminished. One consequence for both sexes is a reduced expression of sexual behaviors [40].

Sexual behavior is also affected by a decreased behavioral responsiveness to steroid hormones. For example, ovariectomized female hamsters housed in LP become sexually receptive if treated sequentially with estradiol and progesterone. In contrast, this treatment is less effective when hamsters have been housed in SP [2,3,7,20,26,28]. Responsiveness of non-reproductive behaviors to sex 
steroid hormones is also influenced by day length. Among female hamsters, the facilitation of locomotor activity by estradiol is attenuated in SP [28], as is the suppression of aggressive behavior by progesterone [20]. Taken together, these data suggest that inhibitory photoperiods induce significant changes in responsiveness of steroid hormone-dependent behaviors.

There are many ways behavioral responsiveness could be modulated by changing photoperiodic conditions. For example, the sequential effects of estradiol and progesterone on lordosis involve their binding to intracellular estrogen (ER) and progestin (PR) receptors, respectively, and subsequent modulation of gene expression [41]. In fact, one critical action of estradiol is to induce the expression of PRs $[9,21]$. Thus, any change in the availability or functional characteristics of steroid receptors could influence the ability of estradiol and/or progesterone to facilitate lordosis [8].

Photoperiodic effects on steroid binding have been examined previously using techniques with less anatomical specificity than immunocytochemistry. For example, binding assays failed to detect photoperiod-induced changes in nuclear $\left[{ }^{3} \mathrm{H}\right]$ estradiol or cytosolic $\left[{ }^{3} \mathrm{H}\right] \mathrm{R} 5020$ binding in the hypothalamus or preoptic area of female hamsters [7] and sheep [6], in cytosolic or nuclear $\left[{ }^{3} \mathrm{H}\right]$ estradiol binding in pooled limbic tissue derived from male hamsters [13], or in the total number of nuclear androgen receptors in the hypothalamus of male hamsters [44], although this latter study did report differences in the number of androgen receptors found in pituitary tissue between LP and SP males. Earlier studies likewise failed to detect photoperiodic effects on steroid binding [5,23]. However, SP exposure might alter steroid receptor numbers within specific regions of the brain without having significant effects in pooled tissue samples containing many different brain regions. The large blocks of tissue used in earlier studies may have masked photoperiodic effects restricted to smaller anatomical regions. Immunocytochemistry, which provides greater anatomical resolution, is better suited to detect localized effects on steroid receptor-containing cells in specific nuclei of the brain.

Support for the possibility that day length might influence the number of cells containing ER in hamster brain is provided by two studies that used daily melatonin injections in ovariectomized females to mimic an inhibitory photoperiod [25,31]. Hamsters administered melatonin each day for 8 [31] or 12 [25] weeks had reduced uterine weights and diminished gonadotropin secretion. Melatonin treatment also decreased the number of ERIR cells in the mPOA [25,31] and bed nucleus of the stria terminalis (BNST) [25] of both ovariectomized as well as gonadally intact hamsters.

In the present experiments, we used an immunocytochemical technique to examine photoperiodic effects on ER and PR in regions of the forebrain which either contain high concentrations of steroid receptors [30,32] or are known to be important for mediating effects of steroid hormones on behavioral or physiological functions related to reproduction [41].

\section{Materials and methods}

\subsection{Animals and housing}

LVG female Syrian hamsters (Mesocricetus auratus) weighing between $80-100 \mathrm{~g}$ were obtained from Charles River Breeding Laboratories (St. Constant, Canada). Animals were housed in hanging wire cages $(17.5 \times 17.5 \times 25$ $\mathrm{cm}$ ) in a 14L:10D cycle (lights on at 0700) and were provided water and Purina Rat Chow (No. 5001) ad libitum.

\subsection{Surgery and pretest}

Beginning one week after arrival, animals were anesthetized with sodium pentobarbital $(80 \mathrm{mg} / \mathrm{kg}$ body weight), supplemented if necessary with Metofane (Methoxyflurane, Pitman-Moore, Mundelein, IL), and ovariectomized.

Animals used for behavioral analyses were given a lordosis pretest two weeks after recovery from surgery in order to assess hormonal responsiveness and to provide sexual experience. Estradiol benzoate (EB; $5 \mu \mathrm{g}$ ) and progesterone (P; $500 \mu \mathrm{g}$ ) were injected subcutaneously 52 and $4 \mathrm{~h}$, respectively, prior to the test. Females were placed in Plexiglas arenas $(30 \times 36 \times 30 \mathrm{~cm})$ and allowed to interact with sexually experienced males for $0.5 \mathrm{~h}$. Females showing lordosis were noted; lordosis was defined as a motionless posture, with back arched and tail raised. Hamsters that were not receptive were brushed on the flank and perineum with an artist's paintbrush to ensure that they received appropriate tactile stimulation. Any hamster still failing to show lordosis was eliminated from the study.

\subsection{Behavior testing}

Hamsters tested for sexual receptivity were hormonally primed and placed alone for $5 \mathrm{~min}$ in the testing arena. Following this adaptation period, adult males with minimal sexual experience were introduced to provide relevant sensory cues, and testing was begun. Tactile stimulation of the females' flank and perineum was also provided by the experimenter using a soft, $1 \mathrm{~cm}$ artist's paint brush during the test period. All tests lasted $3 \mathrm{~min}$; females which displayed lordosis for more than $5 \mathrm{~s}$ were considered behaviorally receptive. For these animals, the time between the introduction of the male and the first display of lordosis (lordosis latency), as well as the cumulative time spent in lordosis (lordosis duration), were recorded. Fe- 
males failing to show lordosis were assigned latency and duration values of 180 and $0 \mathrm{~s}$, respectively.

\subsection{Immunocytochemistry}

The immunocytochemical procedures used to visualize ERIR and PRIR have been described previously [19,32]. For ER, hamsters were killed in the early afternoon with an overdose of sodium pentobarbital $(240 \mathrm{mg} / \mathrm{kg})$. Sodium heparin (5000 $\mathrm{U}$ dissolved in $1.0 \mathrm{ml}$ of $0.15 \mathrm{M} \mathrm{NaCl}$ ) was injected into the left ventricle of the heart, and the right atrium was cut to allow blood and perfusate to exit. Hamsters were then transcardially perfused with $\mathrm{NaCl}(15$ $\mathrm{ml} ; 0.15 \mathrm{M}$ ) followed by $250 \mathrm{ml}$ of $2 \%$ paraformaldehyde with $0.4 \%$ glutaraldehyde in $0.1 \mathrm{M}$ sodium phosphate buffer (flow rate approximately $25 \mathrm{ml} / \mathrm{min}$ at a pressure of $100 \mathrm{mmHg}$ ). Brains were removed and stored overnight in $0.1 \mathrm{M}$ sodium phosphate buffer with $20 \%$ sucrose at $4^{\circ} \mathrm{C}$. The next day, $40 \mu \mathrm{m}$ sections were cut on a freezing microtome and stored in cryoprotectant at $-20^{\circ} \mathrm{C}$ until used for immunocytochemistry (ICC). In each study, all brain tissue was collected prior to the start of ICC, so all sections were processed in a single ICC run. Tissue sections were first rinsed ( 3 times for $5 \mathrm{~min}$ each) in $0.05 \mathrm{M}$ Tris-buffered saline (TBS; pH 7.6), followed by a $10 \mathrm{~min}$ rinse in TBS with $1 \%$ sodium borohydride. Sections were rinsed again in TBS (4 times for 5 min each), followed by a 20 min rinse in TBS with $1 \%$ hydrogen peroxide, $1 \%$ bovine serum albumin, and $20 \%$ normal rabbit serum (NRS). Sections were incubated for $72 \mathrm{~h}$ at $4^{\circ} \mathrm{C}$ in primary antibody (H222; $1 \mu \mathrm{g} / \mathrm{ml}$; Abbott Laboratories, North Chicago, IL) in TBS with $0.5 \%$ Triton X-100, $0.1 \%$ gelatin, $1 \%$ NRS, and $0.02 \%$ sodium azide. Upon removal, sections were rinsed 3 times ( 5 min each) in the same buffer, and incubated for $90 \mathrm{~min}$ at room temperature in biotinylated rabbit anti-rat antibody (Vector Laboratories, Burlingame, CA) at a concentration of $6 \mu \mathrm{g} / \mathrm{ml}$ in the same buffer. Sections were then given three 5 min washes, twice in the same buffer and once in 0.05 M TBS, and incubated for $90 \mathrm{~min}$ at room temperature in the avidinDH-biotinylated horseradish peroxidase-H complex (Vectastain Elite ABC kit, Vector Laboratories, Burlingame, CA) at a concentration of $1: 100$ in $0.05 \mathrm{M}$ TBS. Finally, sections were rinsed twice in $0.05 \mathrm{M}$ TBS with $0.02 \%$ sodium azide and $0.5 \%$ Triton $\mathrm{X}-100$, once in $0.05 \mathrm{M}$ TBS, and were exposed to a chromagen (diaminobenzidine, $0.05 \%$ in TBS with $0.05 \%$ hydrogen peroxide) for approximately 4 min.

The procedures for visualizing PRIR were similar to those used for ERIR except that hamsters were perfused with $2 \%$ acrolein in $0.1 \mathrm{M}$ sodium phosphate buffer. The primary antibody was B39 $(0.1 \mu \mathrm{g} / \mathrm{ml}$; Abbott Laboratories, North Chicago, IL); the same secondary antibody was used at a concentration of $5 \mu \mathrm{g} / \mathrm{ml}$. Hamsters were always sacrificed $48 \mathrm{~h}$ following estradiol benzoate injections.

\subsection{Analysis of steroid receptor immunoreactivity}

All analyses were performed using modifications of image analysis protocols described earlier [1]. IMAGE 1.54 software (W. Rasband, NIH, Bethesda, MD; http://rsb.info.nih.gov/nih-image) on a Macintosh Quadra 700 was used to record and analyze images taken by an MTI CCD72 camera (DAGE MTI, Michigan City, MI) attached to a Leitz Dialux 20 microscope. To adjust camera gain and black levels, a cover-slipped test slide with black marks was used. The marks were assigned a grey-scale value of 255 (maximum) and the background was set between 10-15. These settings were used for all analyses. On slides with tissue to be imaged, the threshold between background and foreground staining was established by determining the average grey-scale value for the area to be analyzed, and setting the threshold either 2 (ERIR) or 4 (PRIR) standard deviations above this average. The minimum and maximum particle size analyzed was 15 and 100 pixels, respectively.

For each anatomical area examined, the number of detectable steroid receptor-containing cells was compared between groups of hamsters housed in different photoperiods. Additionally, the effect of photoperiod on cell staining intensity was analyzed because the intensity of immunostaining is believed related to the number of antibody-receptor interactions within a cell, with more darkly stained cells having more receptors capable of binding antibody. Cells were categorized as lightly (10-89), moderately (90-169), or darkly (170-255) stained, according to the grey scale value of their darkest pixel.

\subsection{Anatomy}

Brain sites known to contain ER and/or PR and potentially relevant to photoperiod-induced alterations in steroid responsiveness were examined. These included the rostral, middle, and caudal VMH, the dorsal and ventral portions of the rostral and caudal mAMYG, the rostral and caudal mPOA, and the ARC (Fig. 1). We also analyzed the mTu, a steroid receptor-rich area lateral to the VMH because effects of genital stimulation on cells in this region have been reported [49]. Single sections from all brains evaluated were matched along their anterior-posterior plane for each anatomical region analyzed, including their separate rostral to caudal, or dorsal to ventral subdivisions. Cells were counted unilaterally and for each brain, the same side was used for all areas. There was little difference in the staining characteristics of one side compared to the other, and unilateral counts reduced medial to lateral variability in the area analyzed.

\subsection{Statistical analysis}

The effects of photoperiod on the proportion of hamsters showing lordosis was determined using Chi-square 

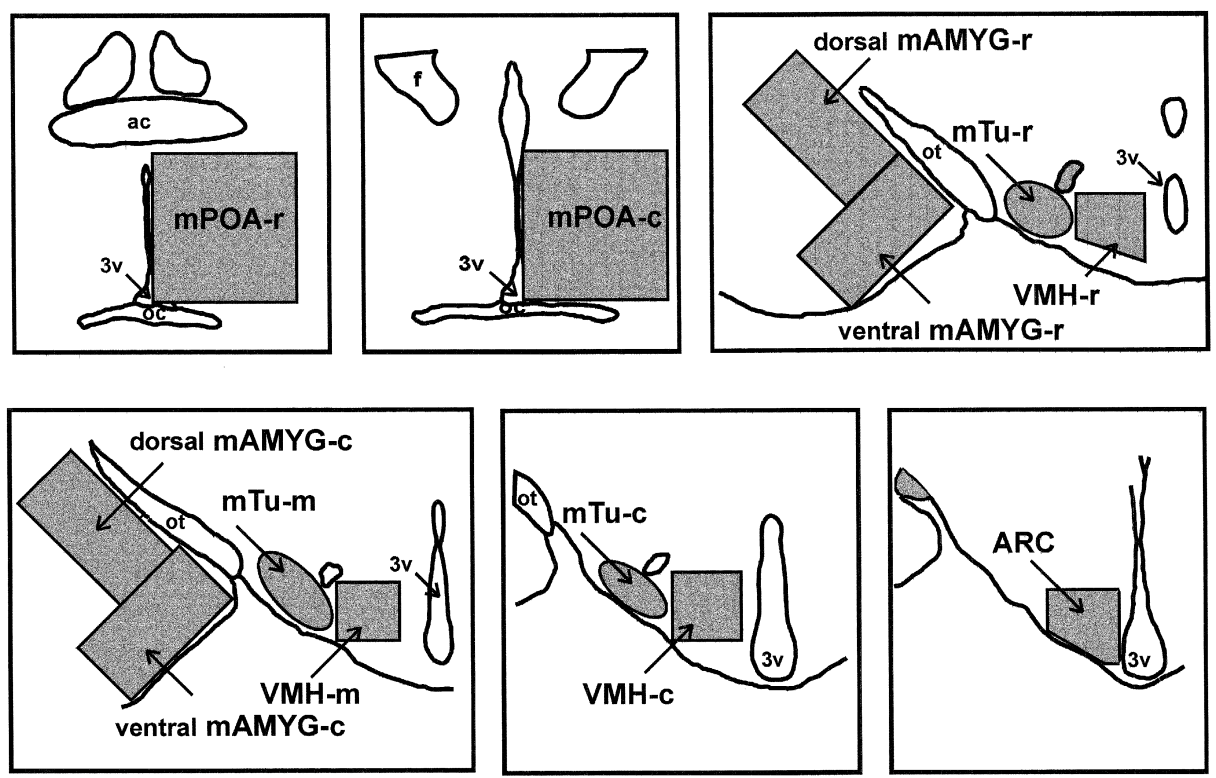

Fig. 1. Brain sites in which cells expressing estrogen or progestin receptor immunoreactivity (ERIR; PRIR) were quantified. For the mPOA, cells were counted within two $1,000,000 \mu \mathrm{m}^{2}$ boxes, one rostral (r); one caudal (c). For the dorsal mAMYG, cells were counted within two (r, c) $500,000 \mu \mathrm{m}^{2}$ rectangles drawn parallel to the optic tract; for the ventral mAMYG, cells were counted within boxes of similar size (r, c) drawn along the base of the brain. For the ARC, cells were counted within one 250,000 $\mu \mathrm{m}^{2}$ box drawn next to the 3rd ventricle. For the VMH, cells were counted within three similar-sized boxes at $\mathrm{r}$, middle $(\mathrm{m})$ and $\mathrm{c}$ levels, all located medial to the fornix. At the same $\mathrm{r}, \mathrm{m}$ and c levels, mTu cells were counted within an area lateral and ventral to the fornix, and medial to the mAMYG. $\mathrm{VMH}=$ ventromedial nucleus of the hypothalamus; mTu $=$ medial tuberal region; $\mathrm{mPOA}=$ medial preoptic area; $\mathrm{mAMYG}=$ medial nucleus of the amygdala; $\mathrm{ARC}=$ arcuate nucleus; $\mathrm{ac}=$ anterior commissure, $\mathrm{f}=$ fornix, $3 \mathrm{v}=3 \mathrm{rd}$ ventricle.

analyses. Independent samples $t$-tests were used to evaluate differences between LP and SP hamsters in lordosis latency and duration, and in the total number of detectable ERIR and PRIR cells within specific brain sites. All $t$-tests were 2-tailed except where noted. The effects of photoperiod on the relative proportion of light, moderate, and darkly stained cells within brain sites were assessed using a $2 \times 3$ mixed design ANOVA with post-hoc independent samples $t$-tests. Results were considered statistically significant if $P<0.05$. All analyses were done with CRISP (Crunch Interactive Statistical Package, version 4, Crunch Software, 1991, Oakland, CA).

\subsection{Procedures}

\subsubsection{Experiment 1}

We evaluated photoperiodic influences on ERIR and sexual receptivity in separate groups of female hamsters. Two weeks after ovariectomy, 57 hamsters were matched for body weight and transferred from 14L:10D to either LP (16L:8D) or SP (8L:16D) conditions (week 0). The midpoints of the new photoperiods were the same as they had been in 14L:10D. On week 10, LP and SP groups were subdivided and scheduled for either behavior testing $(n=$ 36) or immunocytochemical analysis of ERIR $(n=21)$. Hamsters tested for lordosis were injected with $1.6 \mu \mathrm{g}$ EB / $100 \mathrm{~g}$ of body weight, followed $48 \mathrm{~h}$ later by $200 \mu \mathrm{g}$ P. Both hormones were dissolved in sesame oil vehicle.
Four hours after P, lordosis was evaluated (Test 1) at approximately mid-day after which hamsters were returned to their respective photoperiods. On week 11, the same hormonal priming and testing procedures were repeated (Test 2). EB in this experiment was administered on a body weight basis because companion groups (not reported here) were chronically food restricted and had significantly reduced body weights. Hamsters used for immunocytochemical analysis of ERIR were sacrificed between weeks 10 and 11.

\subsubsection{Experiment 2}

We evaluated the effect of photoperiod on both PRIR and sexual receptivity in the same animals. Two weeks after ovariectomy, 24 hamsters were divided into two groups matched for body weight; one remained in LP (14L:10D) and the other was moved to SP (8L:16D) for the next ten weeks. On week 10, all hamsters received 2.5 $\mu \mathrm{g}$ EB, followed $48 \mathrm{~h}$ later by $250 \mu \mathrm{g} \mathrm{P}$; lordosis was tested $4 \mathrm{~h}$ after $\mathrm{P}$ injection. Following this test, animals were returned to their respective photoperiods for another two weeks. On week 12, they were injected with $2.5 \mu \mathrm{g}$ EB then sacrificed $48 \mathrm{~h}$ later and their brains processed for PRIR.

\subsubsection{Experiment 3}

This study further explored the photoperiodic effects on PRIR observed in Experiment 2. Changes in PRIR in SP 
could be due to either central or peripheral effects of photoperiod. Central effects of photoperiod on PRIR could be mediated by a number of mechanisms, such as changes in the ability of estradiol to induce PR synthesis or in post-translational processing of the PR protein. Peripheral effects of SP, however, might lower systemic estradiol levels by altering its metabolism or otherwise interfere with the ability of estradiol to reach the brain. To distinguish between these two general possibilities, we induced PR synthesis in hamsters housed in LP and SP by administering estradiol intracranially, bypassing the systemic circulation and eliminating changes in peripheral estradiol metabolism as a possible factor contributing to the effects of photoperiod on PRIR.

Thirty four female hamsters were ovariectomized; two weeks later they were divided into two groups matched for body weight and placed into LP (14L:10D) or SP (8L:16D). Ten weeks later, bilateral guide cannulas (22 gauge stainless steel) were implanted in the medial basal hypothalamus under sodium pentobarbital anesthesia $(80 \mathrm{mg} / \mathrm{kg}$ body weight). Coordinates were $0.7 \mathrm{~mm}$ caudal to bregma, $\pm 0.75 \mathrm{~mm}$ lateral to the sinus, and $7.7 \mathrm{~mm}$ below dura, with the skull flat between bregma and lambda. Four to five days later, a single inner cannula containing estradiol was lowered into one of the two guide cannulae of each animal; the inner cannula tip projected approximately 0.5 $\mathrm{mm}$ beyond the tip of its guide. Inner cannulae (28 gauge) were prepared by tapping them into crystalline estradiol$17 \beta$ (Sigma, St. Louis, MO) until their tips were completely filled; neither total estradiol by volume nor estradiol released in vivo was measured. Hamsters so treated were killed $48 \mathrm{~h}$ later and their brains processed for evaluating PRIR. The location of the inner cannula tip was determined for each brain, and computer-aided image analysis of PRIR cell number and staining intensity was per- formed on a subset of brains matched for inner cannulae placement. Cannula tips in the brains analyzed were located just dorsal to the $\mathrm{VMH}$ in the middle or caudal sections between the fornix and the 3rd ventricle.

\section{Results}

\subsection{Experiment 1}

\subsubsection{Effect of photoperiod on lordosis}

On Test 1 , fewer than $50 \%$ of the animals from either group showed lordosis; photoperiod failed to affect either the percent responding, lordosis latency, or lordosis duration (Fig. 2). This low level of responding in both groups is likely due to the reduction in hormone responsiveness sometimes observed after prolonged intervals following ovariectomy $[15,16]$. On Test 2 , one week later, the proportion of receptive hamsters increased among LP females but dropped slightly among SP females $\left(\chi^{2}=9.13, P<\right.$ 0.01; Fig. 2). This suggests that estradiol administration prior to test 1 was sufficient to resensitize LP, but not SP, hamsters. When all animals were considered, mean lordosis latency was longer $(t(34)=2.451, P<0.05)$ and mean lordosis duration was shorter $(t(34)=2.637, P<0.05)$ for hamsters in SP (Fig. 2). When only the behaviorally receptive hamsters were compared, these response differences were eliminated. Thus, photoperiod influenced the proportion of hamsters becoming sexually receptive, but had minimal effects on the quality of lordosis in those animals in which receptivity was induced.

\subsubsection{Effect of photoperiod on ERIR}

The anatomical distribution of ERIR we observed is consistent with previous studies using both immunocyto-

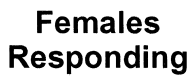

Duration

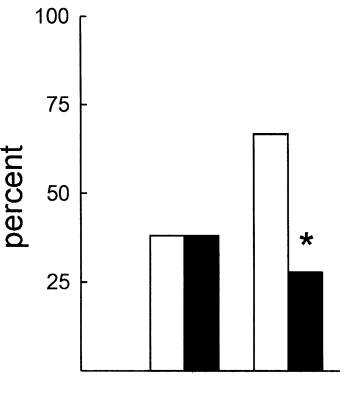

Test1 Test 2

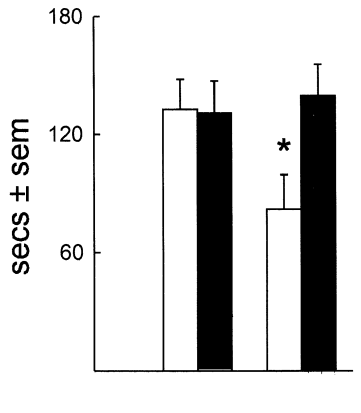

Test1 Test 2

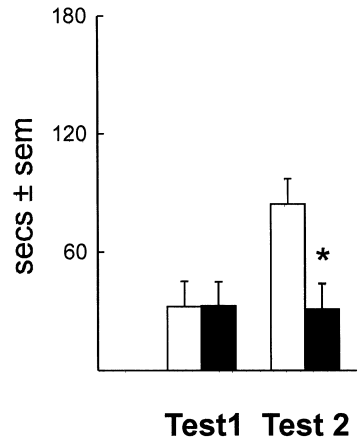

Short Photoperiod

Fig. 2. Experiment 1: Effect of photoperiod on lordosis. The percentage of hamsters showing lordosis for at least $5 \mathrm{~s}$ during a $3 \mathrm{~min}$ test (Females Responding), latency to the first display of lordosis (Latency) and the total time spent in lordosis (Duration) are shown. Latency and duration values (mean seconds \pm S.E.M.) include responders and non-responders. Test 1 was given 10 weeks after beginning photoperiodic treatment; Test 2 occurred one week later. $N=18$ (LP); 18 (SP). Statistically significant differences are indicated by ' $*$ '; $P<0.05$. 


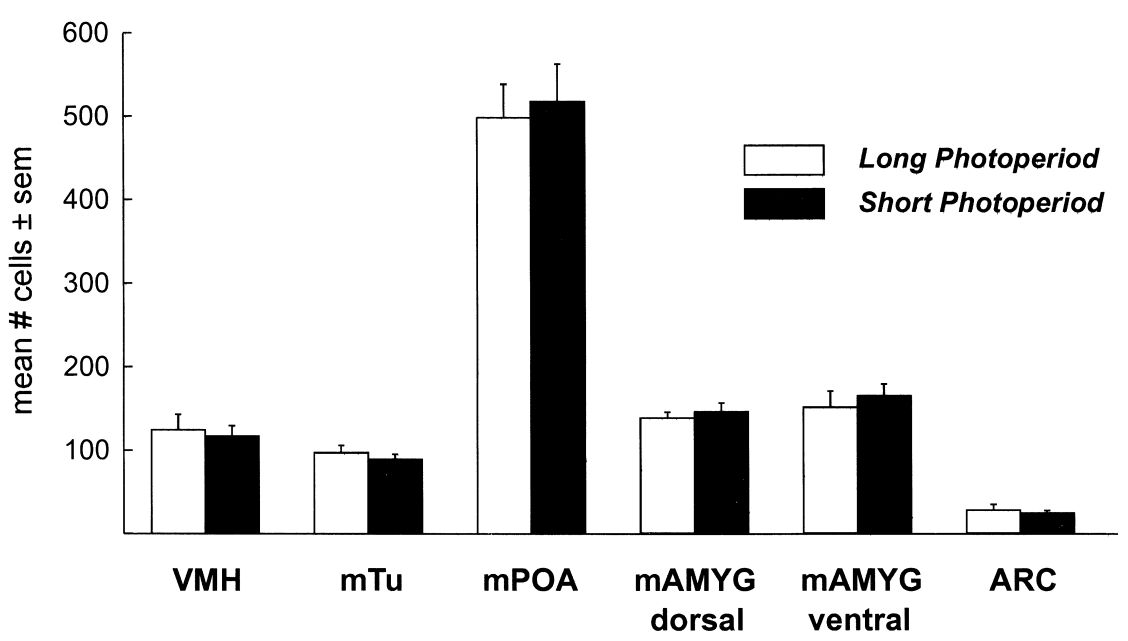

Fig. 3. Experiment 1: Effect of photoperiod on numbers of cells expressing estrogen receptor immunoreactivity (ERIR). Numbers of detectable ERIR cells within each brain site, summed across all rostral to caudal sections analyzed, are depicted (mean total \pm S.E.M.). No effect of photoperiod on total ERIR cell numbers was observed. $N=8$ (LP); 11 (SP).

chemistry and steroid autoradiography in female Syrian hamsters [30,32]. The mPOA, immediately adjacent to the third ventricle, contained the densest population of labelled cells. Staining was also seen in the VMH, mTu, ARC, and
mAMYG. Photoperiodic treatment generally had no effect on either the number of cells containing ERIR (Fig. 3) or their relative staining intensity (Table 1), with two exceptions. Animals in SP had a greater number of ERIR cells in

Table 1

Photoperiodic effects on neural estrogen receptor immunoreactivity among female hamsters

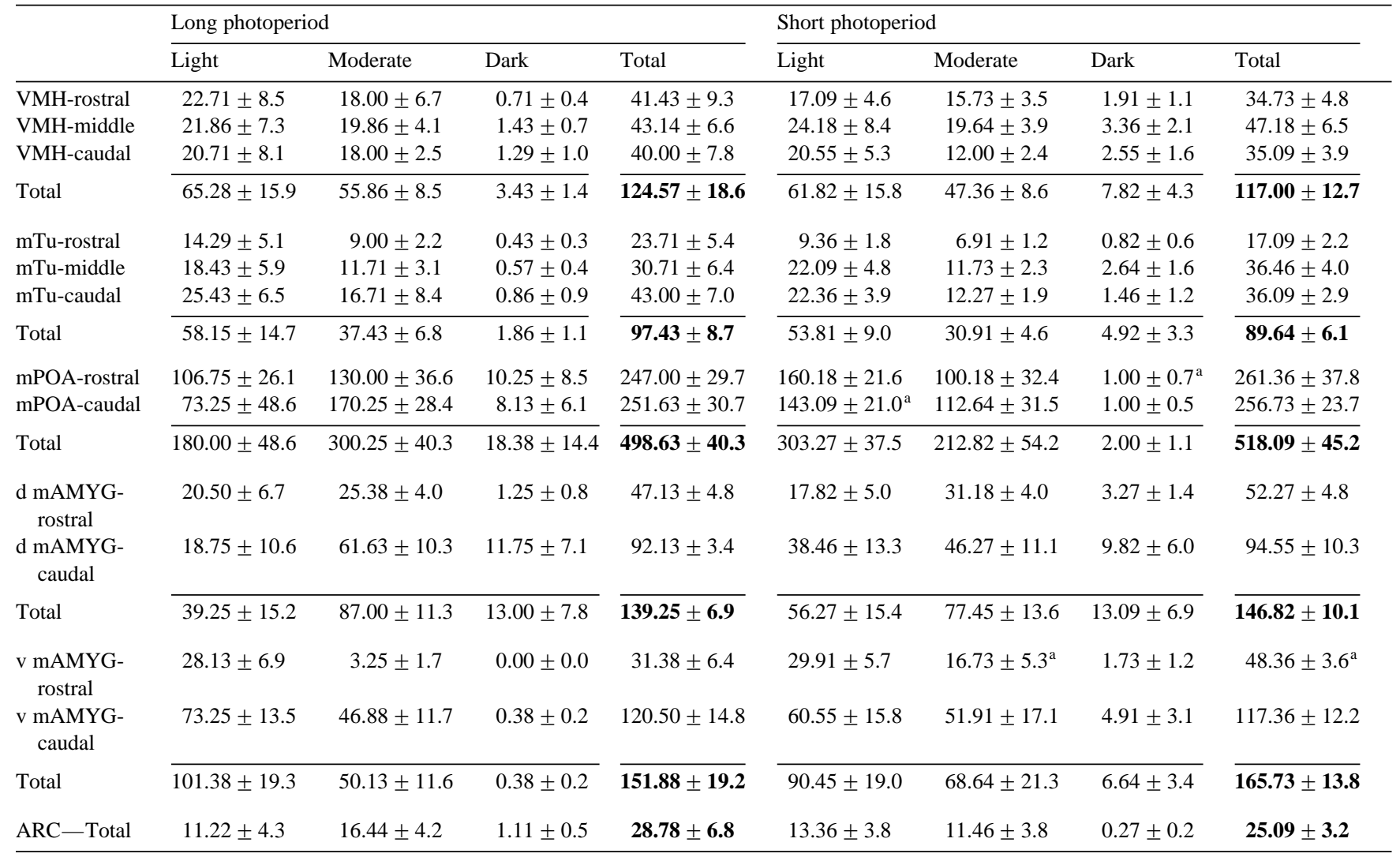

${ }^{a}$ Significantly different from corresponding Long Photoperiod value, $P<0.05$.

Mean number \pm S.E.M. of lightly (1-89), moderately (90-169), and darkly (170-254) stained estrogen receptor immunoreactive cells, based on the gray scale value of their darkest pixel, within each anatomical region examined. The total number of cells within each pixel density range, as well as the total number of cells within each regional subdivision, are also shown. Numbers in boldface represent the regional subdivisions combined. $N=8$ (LP); 11 (SP). 
Females

Responding

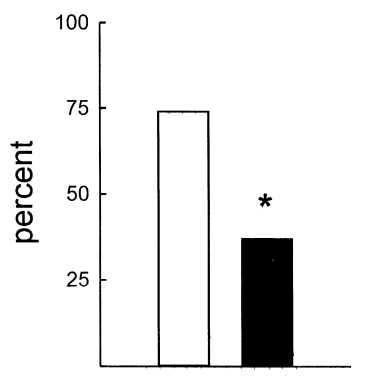

Latency



Duration

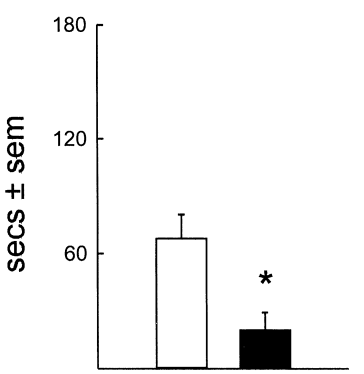

\section{Long Photoperiod}

\section{Short Photoperiod}

Fig. 4. Experiment 2: Effect of photoperiod on lordosis. The percent of hamsters showing lordosis for at least $5 \mathrm{~s}$ during a $3 \mathrm{~min}$ test (Females Responding), latency to the first display of lordosis (Latency) and the total time spent in lordosis (Duration) are shown. Latency and duration values (mean seconds \pm S.E.M.) include responders and non-responders. $N=19$ (LP); 19 (SP). Statistically significant differences are indicated by ' $*$ '; $P<0.05$.

the ventral subdivision of the rostral mAMYG $(t(17)=$ 2.477, $P<0.05$; Table 1). Also, photoperiod had a modest but opposite influence on the staining intensity of ERIR cells in the mPOA, particularly its caudal component. Exposure to SP increased the number of lightly stained cells (gray scale value 1-89) and decreased the number of moderately stained cells (gray scale value 90-169), although this effect was not statistically significant (Table 1).

\subsection{Experiment 2}

\subsubsection{Effect of photoperiod on lordosis}

Exposure to SP significantly reduced the percentage of females exhibiting lordosis $\left(\chi^{2}=5.216, P<0.05\right.$; Fig. 4). Lordosis latency was longer $(t(36)=2.321, P<0.05)$ and lordosis duration shorter $(t(36)=2.742, \quad P<0.01)$ among hamsters in SP, replicating the results of Experiment 1, Test 2. As in Experiment 1, these latency and duration effects were not present when only receptive females were compared.

\subsubsection{Effect of photoperiod on PRIR}

PRIR was detected in the mPOA, VMH, mTu, and ARC. Very faint staining was also observed in the dorsal, but not in the ventral mAMYG. In the VMH, mPOA, mAMYG and the ARC, exposure to SP significantly reduced the total number of PRIR cells detected (Fig. 5) and diminished their staining intensity (Table 2). In the mTu, staining intensity but not total cell count was similarly affected.

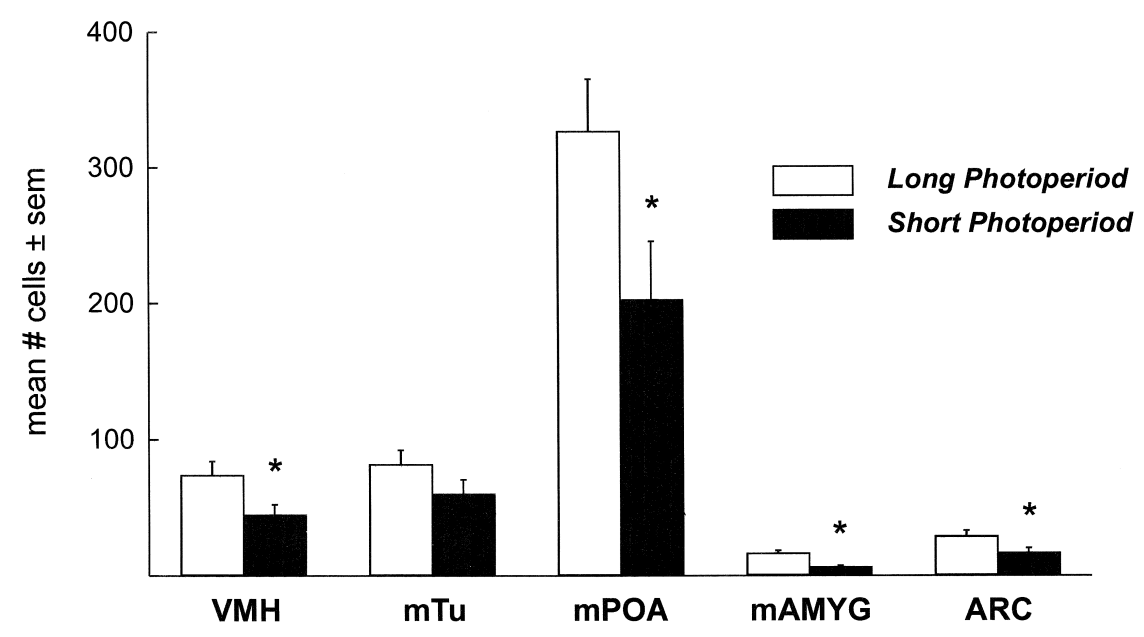

Fig. 5. Experiment 2: Effect of photoperiod on numbers of cells expressing progestin receptor immunoreactivity (PRIR). Numbers of detectable PRIR cells within each brain site, summed across all rostral to caudal sections analyzed, are depicted (mean total \pm S.E.M.). All animals were administered estradiol benzoate systemically, $48 \mathrm{~h}$ prior to sacrifice. Short photoperiod hamsters had significantly fewer cells in the VMH, mPOA, mAMYG, and ARC. $N=11$ (LP); 12 (SP). Statistically significant differences are indicated by ' $*$ '; $P<0.05$. 
Table 2

Photoperiodic effects on neural progestin receptor immunoreactivity among female hamsters administered estradiol systemically

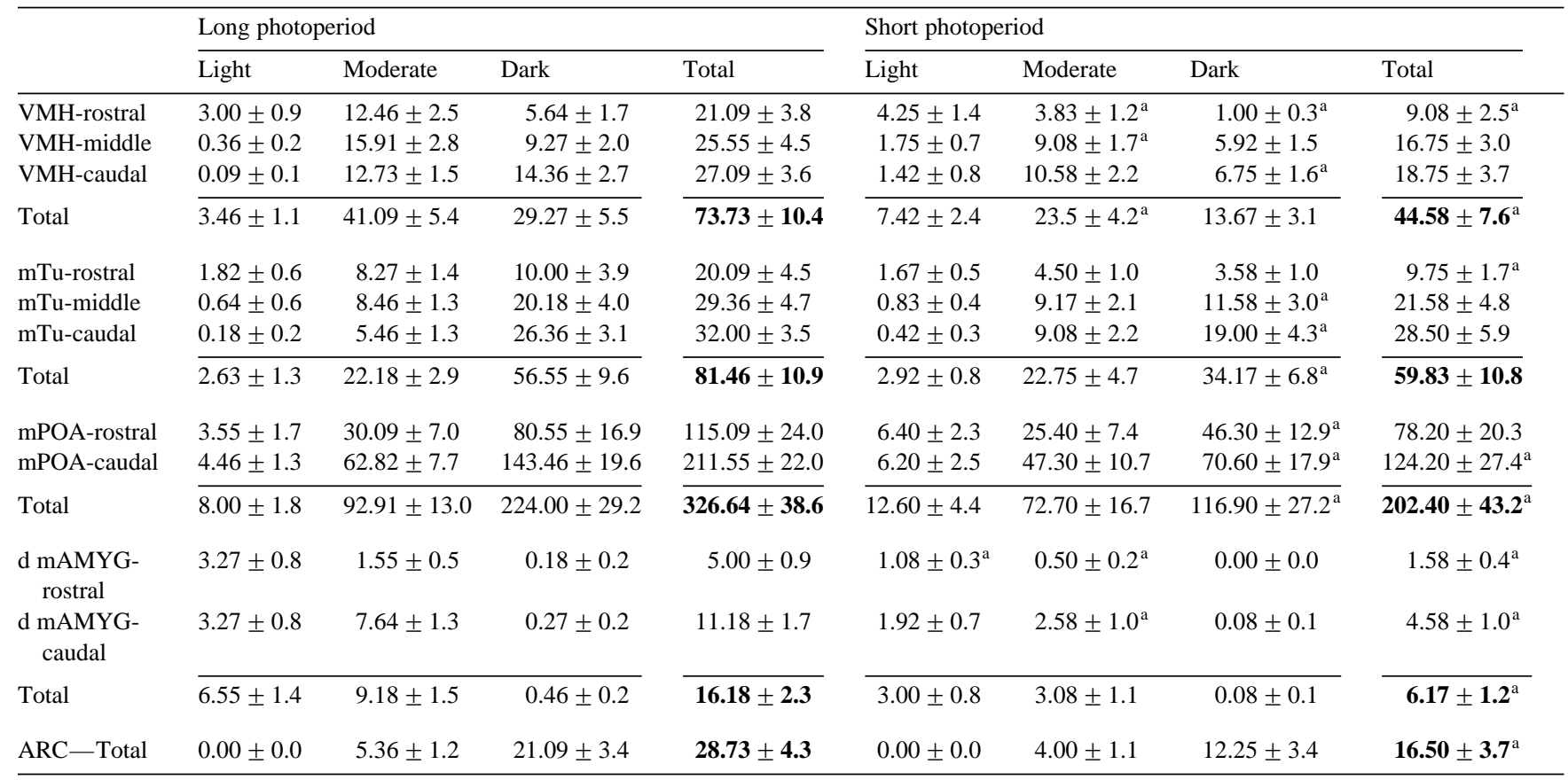

${ }^{a}$ Significantly different from corresponding Long Photoperiod value, $P<0.05$.

Mean number \pm S.E.M. of lightly (1-89), moderately (90-169), and darkly (170-254) stained progestin receptor immunoreactive cells, based on the gray scale value of their darkest pixel, within each anatomical region examined. The total number of cells within each pixel density range, as well as the total number of cells within each regional subdivision, are shown. Numbers in boldface represent the regional subdivisions combined. $N=11$ (LP); 12 (SP).

\subsection{Experiment 3}

\subsubsection{Effect of photoperiod on PRIR}

Estradiol implanted unilaterally into the medial basal hypothalamus induced PRIR bilaterally in the VMH, mTu, and mPOA although staining was darker on the side ipsilateral to the implant. Cells were counted only on the side ipsilateral to the estradiol implant. Exposure to SP significantly reduced the number of PRIR cells in the VMH (Fig. 6), and diminished PRIR staining intensity in both the VMH and mPOA (Table 3). The number of PRIR cells was reduced in the mTu, but this effect was not significant. Analysis of rostral to caudal subdivisions within the brain areas analyzed indicated that SP effects were

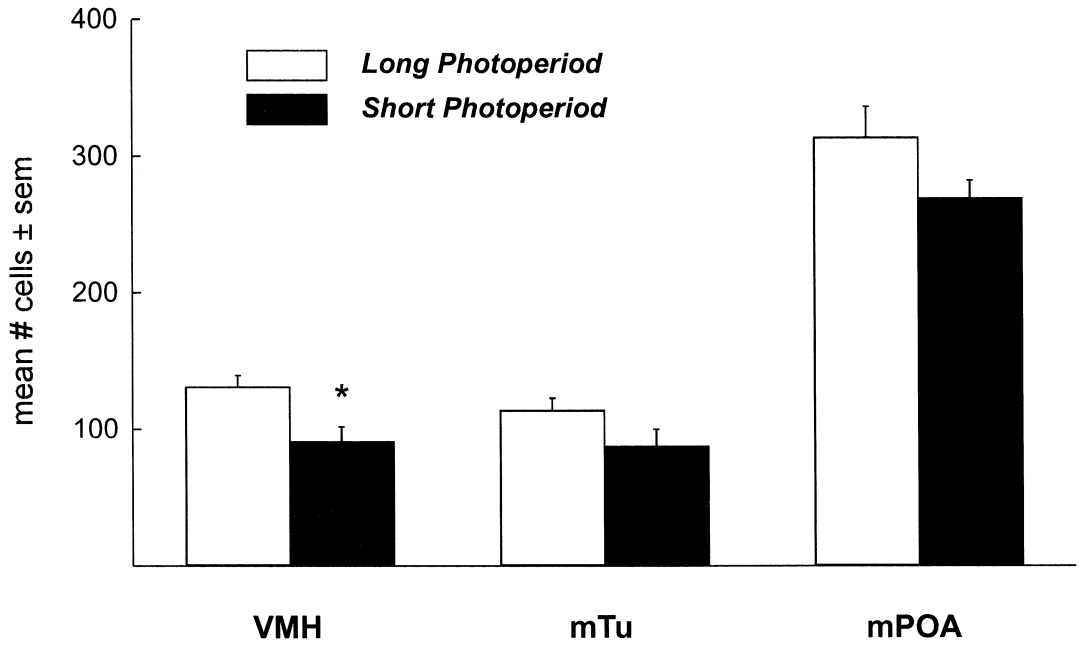

Fig. 6. Experiment 3: Effect of photoperiod on numbers of cells expressing progestin receptor immunoreactivity (PRIR). All animals were administered estradiol via unilateral mediobasal hypothalamic cannulae, $48 \mathrm{~h}$ prior to sacrifice. Numbers of detectable PRIR cells within each brain site, summed across all rostral to caudal sections analyzed, are depicted (mean total \pm S.E.M.). Hamsters in the short photoperiod had fewer cells in the VMH. $N=5$ (LP); 5 (SP), matched for cannulae placement. Statistically significant differences are indicated by ' $*$ '; $P<0.05$. 
Table 3

Photoperiodic effects on progestin receptor immunoreactivity among female hamsters administered estradiol in the medial basal hypothalamus

\begin{tabular}{|c|c|c|c|c|c|c|c|c|}
\hline & \multicolumn{4}{|c|}{ Long photoperiod } & \multicolumn{4}{|c|}{ Short photoperiod } \\
\hline & Light & Moderate & Dark & Total & Light & Moderate & Dark & Total \\
\hline VMH-rostral & $0.00 \pm 0.0$ & $20.40 \pm 3.2$ & $19.60 \pm 8.1$ & $40.00 \pm 8.6$ & $2.20 \pm 1.6$ & $17.60 \pm 2.6$ & $9.80 \pm 3.2$ & $29.60 \pm 4.8$ \\
\hline VMH-caudal & $0.60 \pm 0.4$ & $18.00 \pm 1.7$ & $22.60 \pm 1.9$ & $41.20 \pm 2.1$ & $2.20 \pm 1.3$ & $15.20 \pm 2.7$ & $8.60 \pm 2.8^{\mathrm{a}}$ & $26.00 \pm 4.8^{\mathrm{a}}$ \\
\hline Total & $1.00 \pm 0.6$ & $65.60 \pm 6.7$ & $64.20 \pm 10.9$ & $130.80 \pm 8.8$ & $8.20 \pm 3.8$ & $52.20 \pm 5.7$ & $30.20 \pm 8.7^{\mathrm{a}}$ & $90.60 \pm 11.1^{a}$ \\
\hline mTu-caudal & $0.00 \pm 0.0$ & $10.60 \pm 2.7$ & $33.60 \pm 2.2$ & $44.20 \pm 2.9$ & $0.80 \pm 0.6$ & $8.20 \pm 1.2$ & $20.60 \pm 8.8$ & $29.60 \pm 9.2$ \\
\hline Total & $1.60 \pm 0.9$ & $38.60 \pm 4.9$ & $73.40 \pm 9.9$ & $\overline{113.60 \pm 9.2}$ & $1.80 \pm 1.0$ & $31.40 \pm 1.6$ & $54.20 \pm 11.4$ & $87.40 \pm 12.5$ \\
\hline mPOA-rostral & $0.60 \pm 0.4$ & $53.20 \pm 8.5$ & $92.00 \pm 15.0$ & $145.80 \pm 19.9$ & $3.40 \pm 2.5$ & $66.00 \pm 6.4$ & $50.00 \pm 10.0^{\mathrm{a}}$ & $119.40 \pm 12.6$ \\
\hline mPOA-caudal & $2.80 \pm 2.8$ & $55.80 \pm 5.5$ & $108.80 \pm 21.5$ & $167.40 \pm 13.2$ & $2.00 \pm 1.4$ & $79.60 \pm 8.9^{\mathrm{a}}$ & $67.80 \pm 18.5$ & $149.40 \pm 13.2$ \\
\hline Total & $3.40 \pm 2.7$ & $109.00 \pm 14.0$ & $200.80 \pm 26.9$ & $\overline{313.20 \pm 22.7}$ & $\overline{5.40 \pm 3.7}$ & $145.60 \pm 14.5$ & $117.80 \pm 18.8^{\mathrm{a}}$ & $\overline{268.80 \pm 13.3}$ \\
\hline
\end{tabular}

${ }^{\text {a }}$ Significantly different from corresponding Long Photoperiod value, $P<0.05$.

Mean number \pm S.E.M. of lightly (1-89), moderately (90-169), and darkly (170-254) stained progestin receptor immunoreactive cells, based on the gray scale value of their darkest pixel, within each anatomical region examined. The total number of cells within each pixel density range, as well as the total number of cells within each regional subdivision, are shown. Numbers in boldface represent the regional subdivisions combined. $N=5$ (LP); 5 (SP).

generally present at all levels, although in some cases significant effects were restricted to one subdivision only (Table 3).

\section{Discussion}

Our findings indicate that exposure to a short photoperiod both attenuated sexual receptivity and reduced the number of PR-containing cells in the brain. In contrast, these same conditions had minimal effects on the number of ER-containing cells. In both Experiments 1 and 2, significantly fewer hamsters exposed to SP exhibited lordosis. However, among those females that became receptive, photoperiodic conditions did not affect lordosis latency or duration (Figs. 2 and 4). Immunocytochemical analyses indicated that the major effect we observed on steroid receptors was a decrease among SP hamsters in the number of cells containing PRIR (Fig. 5) and a reduction in cell staining intensity (Table 2). Effects on ERIR when present were subtle in comparison (Fig. 3).

The number of detectable ERIR cells was influenced significantly by photoperiod only in the ventral portion of the rostral mAMYG; SP females had more ERIR cells overall with most falling in the moderately stained category (Table 1). Although this change is consistent with the increased sensitivity of SP females to negative feedback influences of estradiol, the medial amygdala has not been implicated in this effect. However, the arcuate nucleus does seem involved [35], yet photoperiodic conditions did not affect ERIR in this region (Table 1). Thus, the functional significance of increased ERIR in the rostral ventral mAMYG is unclear.

SP conditions had only marginal effects on ERIR in the mPOA; the number of moderately and darkly stained cells was lower, accompanied by an increase in the number of lightly stained cells (Table 1). This suggestion of fewer ER in mPOA cells among SP hamsters is consistent with earlier findings $[25,31]$ in which mPOA ERIR was significantly diminished. However, hamsters in these earlier studies received exogenous melatonin with amplitude and nightly duration characteristics which markedly exceeded those resulting from exposure to SP, the condition used in the present study [38]. Thus, non-physiological melatonin treatment in part could have been responsible for the observed reduction in mPOA ERIR.

In contrast to the relatively subtle effects of photoperiod on ERIR that we observed, exposure to SP did reduce PRIR cell number and staining intensity in the brain regions examined (Experiment 2; Fig. 5, Table 2). In evaluating these effects, it is important to note that with our ICC protocol, constitutively expressed PRs are not observed; treatment with estradiol is required to induce PR expression. Consequently, significantly diminished PR expression among hamsters in SP might result from a decreased responsiveness to estradiol or to a diminished availability of this hormone, due perhaps to changes in its peripheral clearance rate. However, we have ruled out the latter possibility; radioimmunoassay of estradiol $48 \mathrm{~h}$ after injections of EB suggested that circulating levels were not significantly different between females housed in LP or SP conditions (unpublished observations). In addition, when estradiol was applied directly to the medial basal hypothalamus through an intracranial implant, SP females expressed significantly reduced PRIR in both the VMH and the mPOA (Fig. 6, Table 3). Together, these results suggest that exposure to SP can both attenuate sexual receptivity in response to estradiol and progesterone treatment and impair PR induction by estradiol. Because the latter effect was observed with both systemic and intracranial adminis- 
tration of estradiol, photoperiodic effects on lordosis and PRIR were not likely dependent on reduced circulating levels of estradiol.

The photoperiodic effect we observed of particular relevance to the lordosis response was the reduction in the number of cells containing PRIR in the VMH. Although quantitatively this change was noticeably smaller than the change observed in mPOA, its importance to sexual behavior may be clearer. Numerous studies in rats and hamsters have demonstrated that the VMH is a critical neural site for the hormonal induction of sexual receptivity $[18,37,41,47]$. In hamsters, the progesterone that follows estradiol priming must act in both the VMH and ventral tegmental area (VTA) to induce an optimal lordosis response $[17,42]$. While progesterone may act in the VTA via a membrane receptor, estradiol-induced, intracellular PRs are essential in the VMH [17]. Thus, the decreased number of cells expressing PRIR in the VMH of SP females would be expected to impede the hormonal induction of lordosis.

In addition to the reductions in PRIR in the hamster $\mathrm{VMH}$, photoperiodic effects were also observed in the mPOA, mAMYG, mTu and ARC. Sex steroid hormone receptors in these regions mediate a number of hormonal effects on behavioral and physiological processes that may be influenced by SP conditions. These include sexual and aggressive behavior [20,22,39,43], gonadotropin secretion $[24,45,48,50]$, chemosensory processing $[10,27,51,52]$, and locomotor activity [28]. Reduced numbers of cells expressing PRs might contribute to photoperiodic influences on these behavioral and physiological processes.

It may seem paradoxical that SP influenced PRIR in regions where ERIR was not significantly affected because of the necessity for estradiol priming in order to visualize PRs with our ICC protocol. Fewer detectable PRIR cells in the mPOA (Fig. 5) is consistent with the diminished mPOA ER staining intensity observed in Experiment 1 (Table 1), but this effect on ERIR was not present in other regions (VMH, mTu, ARC, mAMYG) where PRIR was reduced in SP (Tables 2 and 3). In fact, the only other change in ERIR, an increase among SP hamsters, occurred in the ventral portion of the rostral mAMYG, a region where no PRIR was detected. Nevertheless, photoperiodic effects on PRIR could still be due to altered neural responsiveness to estradiol. Because induction of the PR protein is a consequence of estradiol action, regardless of the concurrent availability of progesterone to interact with its receptor, it is likely that the decrease in PRIR in SP is due to a change in processes by which estrogen receptors, bound to their ligand, initiate transcription of the PR gene. These could include photoperiod-induced changes in ER conformation which decrease transcriptional activity without altering hormone or antibody binding, or alterations in the availability or activity of co-regulators that are necessary for activation of the PR gene as well as other genes involving sex behavior and/or locomotor activity [29].
Alternatively, the changes we observed in PRIR may be unrelated to effects of photoperiod on responsiveness to estradiol. For example, SP could reduce PR numbers by altering the post-transcriptional processing of the PR protein, or by reducing its half-life.

The discrepancy between changes in ERIR and PRIR in the same part of the brain has precedent. Food depriving hamsters for $48 \mathrm{~h}$ reduces the lordosis response induced by estradiol and progesterone. This effect is accompanied by reduced ERIR in the VMH with no change in PRIR. In the mPOA, ERIR is actually increased while PRIR is decreased $[19,33]$. These findings illustrate two important points. First, lordosis is a complex response, influenced by many hormonal and sociosexual cues, interacting with many areas of the brain. Thus, although food deprivation and SP exposure both inhibit sex behavior and alter neural responses to sex steroids, these manipulations most likely achieve their similar effects by quite different means. Second, although responsiveness to estradiol can influence the induction of PRs, detectable changes in ERIR are neither required nor sufficient to induce changes in PRIR. This may be because experimental manipulations which affect the induction of PRs by estradiol can result from changes which occur downstream from the binding of estradiol to its receptor. Alternatively, this may reflect heterogeneity in the phenotypic characteristics of ER- and PR-containing cells within a given neural site. Thus, even though ERIR might be altered within a specific region, the subset of ERIR-containing cells which contain PR might not be influenced the same way, producing different effects on ERIR and PRIR.

Although it is tempting to associate the SP-induced decrease in sexual receptivity with the decrease in PRIR in the experiments presented here, somatosensory stimulation is also required to trigger the lordosis reflex, and other peripheral cues undoubtedly contribute to the initiation and maintenance of female hamster sexual receptivity [14]. Alterations in responsiveness to any of these external stimuli could contribute to the effects of SP on lordosis.

In summary, our findings suggest that exposure to SP reduces the ability of steroid hormones to induce sexual receptivity. Exposure to SP also causes a decrease in the number of cells expressing PRIR in the VMH, mTu, mPOA, mAMYG, and ARC. In contrast, SP conditions have minimal influence on the numbers of cells expressing ERIR. Control of steroid hormone receptor numbers may provide one mechanism by which short photoperiods affect reproductive physiology, behavior, and other aspects of seasonal energy regulation.

\section{Acknowledgements}

The authors wish to gratefully acknowledge Robin Lempicki for assistance with the immunocytochemistry, and Jay Alexander for assistance with surgical procedures. 
Research supported by HD 30372 to J.B.P.; NS19327 and Senior Scientist Award MH01312 to J.D.B.

\section{References}

[1] A.P. Auger, J.D. Blaustein, Progesterone enhances an estradiol-induced increase in fos immunoreactivity in localized regions of female rat forebrain, J. Neurosci. 15 (1995) 2272-2279.

[2] L.L. Badura, A.A. Nunez, Photoperiodic modulation of sexual and aggressive behavior in female golden hamsters (Mesocricetus auratus): role of the pineal gland, Horm. Behav. 23 (1989) 27-42.

[3] L.L. Badura, W.R. Yant, A.A. Nunez, Photoperiodic modulation of steroid-induced lordosis in golden hamsters, Physiol. Behav. 40 (1987) 551-554.

[4] T.J. Bartness, G.N. Wade, Photoperiodic control of seasonal body weight cycles in hamsters, Neurosci. Biobehav. Rev. 9 (1985) 599-612.

[5] M.J. Baum, P.M.J. Schretlen, Cytoplasmic binding of oestradiol-17b in several brain regions, pituitary, and uterus of ferrets ovariectomized while in or out of oestrus, J. Reprod. Fertil. 55 (1979) 317-321.

[6] E.L. Bittman, J.D. Blaustein, Effects of day length on sheep neuroendocrine estrogen and progestin receptors, Am. J. Physiol. 258 (1990) R135-R142.

[7] E.L. Bittman, C.M. Hegarty, M.Q. Layden, J.A. Jonassen, Influences of photoperiod on sexual behaviour, neuroendocrine steroid receptors and adenohypophysial hormone secretion and gene expression in female golden hamsters, J. Mol. Endocrinol. 5 (1990) 15-25.

[8] J.D. Blaustein, D.H. Olster, Gonadal steroid hormone receptors and social behaviors, Adv. Comp. Environ. Physiol. 3 (1989) 31-104.

[9] J.D. Blaustein, J.C. Turcotte, Estradiol-induced progestin receptor immunoreactivity is found only in estrogen receptor-immunoreactive cells in guinea pig brain, Neuroendocrinology 49 (1989) 454-461.

[10] S.C. Bressler, M.J. Baum, Sex comparison of neuronal fos immunoreactivity in the rat vomeronasal projection circuit after chemosensory stimulation, Neuroscience 71 (1996) 1063-1072.

[11] F.H. Bronson, Mammalian Reproductive Biology, Univ. of Chicago Press, Chicago, 1989.

[12] F.H. Bronson, P.D. Heideman, Seasonal regulation of reproduction in mammals, in: E. Knobil, J.D. Neill (Eds.), The Physiology of Reproduction, Raven Press, New York, 1994, pp. 541-583.

[13] G.V. Callard, P. Mak, D.J. Solomon, Effects of short days on aromatization and accumulation of nuclear estrogen receptors in the hamster brain, Biol. Reprod. 35 (1986) 282-291.

[14] C.S. Carter, Female sexual behavior, in: H.I. Siegel (Ed.), The Hamster: Reproduction and Behavior, Plenum, New York, 1985, pp. 173-189.

[15] C.R. Clark, N.J. MacLusky, B. Parsons, F. Naftolin, Effects of estrogen deprivation on brain estrogen and progestin receptor levels and activation of female sexual behavior, Horm. Behav. 15 (1981) 289-298.

[16] J.A. Czaja, P.C. Butera, Behavioral consequences of hormonal deprivation on the responsiveness of female rats to estradiol, Physiol. Behav. 35 (1985) 873-877.

[17] J.F. DeBold, C.A. Frye, Genomic and non-genomic actions of progesterone in the control of female hamster sexual behavior, Horm. Behav. 28 (1994) 445-453.

[18] J.F. DeBold, C.A. Frye, Progesterone and the neural mechanisms of hamster sexual behavior, Psychoneuroendocrinology 19 (1994) 563579.

[19] Y. Du, G.N. Wade, J.D. Blaustein, Effects of food deprivation on induction of neural progestin receptors by estradiol in Syrian hamsters, Am. J. Physiol. 39 (1996) R978-R983.

[20] A.S. Elliott, A.A. Nunez, Photoperiod modulates the effects of steroids on sociosexual behaviors of hamsters, Physiol. Behav. 51 (1992) 1189-1193.

[21] I.G. Fraile, D.W. Pfaff, B.S. McEwen, Progestin receptors with and without estrogen induction in male and female hamster brain, Neuroendocrinology 45 (1987) 487-491.

[22] J.W. Garrett, C.S. Campbell, Changes in social behavior of the male golden hamster accompanying photoperiodic changes in reproduction, Horm. Behav. 14 (1980) 303-318.

[23] J.D. Glass, R.P. Amann, T.M. Nett, Effects of season and sex on the distribution of cytosolic estrogen receptors within the brain and the anterior pituitary gland of sheep, Biol. Reprod. 30 (1984) 894-902.

[24] B.D. Goldman, S. Brown, Sex differences in serum LH and FSH patterns in hamsters exposed to short photoperiods, J. Steroid Biochem. 11 (1979) 531-538.

[25] S.M. Hill, L.L. Spriggs, N.O. Lawson, R.E. Harlan, Effects of melatonin on estrogen receptor expression in the forebrain of outbred (Lak:LVG) golden hamsters, Brain Res. 742 (1996) 107-114.

[26] G.I. Honrado, L. Paclik, A.S. Fleming, The effects of short day exposure on seasonal and circadian reproductive rhythms of female golden hamsters, Physiol. Behav. 50 (1991) 357-364.

[27] M.A. Joppa, R.L. Meisel, M.A. Garber, c-fos Expression in female hamster brain following sexual and aggressive behaviors, Neuroscience 68 (1995) 783-792.

[28] J.D. Karp, J.B. Powers, Photoperiodic and pineal influences on estrogen-stimulated behaviors in female Syrian hamsters, Physiol. Behav. 54 (1993) 19-28.

[29] J.A. Katzenellenbogen, B.W. O'Malley, B.S. Katzenellenbogen, Tripartite steroid hormone receptor pharmacology: interaction with multiple effector sites as a basis for the cell- and promoter-specific action of these hormones, Mol. Endocrinol. 10 (1996) 119-131.

[30] M.S. Krieger, J.I. Morrell, D.W. Pfaff, Autoradiographic localization of estradiol-concentrating cells in the female hamster brain, Neuroendocrinology 22 (1976) 193-205.

[31] N.O. Lawson, B.E.F. Wee, D.E. Blask, C.G. Castles, L.L. Spriggs, S.M. Hill, Melatonin decreases estrogen receptor expression in the medial preoptic area of inbred (LSH/SsLak) golden hamsters, Biol. Reprod. 47 (1992) 1082-1090.

[32] H.-Y. Li, J.D. Blaustein, G.J. De Vries, G.N. Wade, Estrogen-receptor immunoreactivity in hamster brain-preoptic area, hypothalamus and amygdala, Brain Res. 631 (1993) 304-312.

[33] H.-Y. Li, G.N. Wade, J.D. Blaustein, Manipulations of metabolic fuel availability alter estrous behavior and neural estrogen receptor immunoreactivity in Syrian hamsters, Endocrinology 135 (1994) 240-247.

[34] R.D. Lisk, The estrous cycle, in: H.I. Siegel (Ed.), The Hamster: Reproduction and Behavior, Plenum, New York, 1985, pp. 23-51.

[35] R.D. Lisk, D.S. Ferguson, Neural localization of estrogen-sensitive sites for inhibition of ovulation in the golden hamster Mesocricetus auratus, Neuroendocrinology 12 (1973) 157-160.

[36] A.S.I. Loudon, Photoperiod and the regulation of annual and circannual cycles of food intake, Proc. Nutr. Soc. 53 (1994) 495-507.

[37] C.W. Malsbury, M.O. Miceli, C.W. Scouten, Neural basis of reproductive behavior, in: H.I. Siegel (Ed.), The Hamster: Reproduction and Behavior, Plenum, 1985, pp. 229-259.

[38] E.S. Maywood, M.H. Hastings, M. Max, E. Ampleford, M. Menaker, A.S.I. Loudon, Circadian and daily rhythms of melatonin in the blood and pineal gland of free-running and entrained Syrian hamsters, J. Endocrinol. 136 (1993) 65-73.

[39] M. Miernicki, M.W. Pospichal, J.B. Powers, Short photoperiods affect male hamster sociosexual behaviors in the presence and absence of testosterone, Physiol. Behav. 47 (1990) 95-106.

[40] R.J. Nelson, L.L. Badura, B.D. Goldman, Mechanisms of seasonal cycles of behavior, Annu. Rev. Psychol. 41 (1990) 81-108.

[41] D.W. Pfaff, S. Schwartz-Giblin, M.M. McCarthy, L.-M. Kow, Cellular and molecular mechanisms of female reproductive behaviors, in: E. Knobil, J.D. Neill (Eds.), The Physiology of Reproduction, Raven Press, New York, 1994, pp. 107-220. 
[42] E.T. Pleim, C.A. Lisciotto, J.F. DeBold, Facilitation of sexual receptivity in hamsters by simultaneous progesterone implants into the VMH and ventral mesencephalon, Horm. Behav. 24 (1990) $139-151$.

[43] J.B. Powers, E.A. Steel, J.B. Hutchison, M.H. Hastings, J. Herbert, A.P. Walker, Photoperiodic influences on sexual behavior in male Syrian hamsters, J. Biol. Rhythms 4 (1989) 61-78.

[44] G.S. Prins, A. Bartke, R.W. Steger, Influence of photoinhibition, photostimulation and prolactin on pituitary and hypothalamic nuclear androgen receptors in the male hamster, Neuroendocrinology 52 (1990) 511-516.

[45] R.F. Seegal, B.D. Goldman, Effects of photoperiod on cyclicity and serum gonadotropins in the Syrian hamster, Biol. Reprod. 12 (1975) 223-231.

[46] R.W. Steger, K. Matt, A. Bartke, Neuroendocrine regulation of seasonal reproductive activity in the male golden hamster, Neurosci. Biobehav. Rev. 9 (1985) 191-201.

[47] L.K. Takahashi, Hormonal regulation of sociosexual behavior in female mammals, Neurosci. Biobehav. Rev. 14 (1990) 403-413.
[48] L. Tamarkin, J.S. Hutchison, B.D. Goldman, Regulation of serum gonadotropins by photoperiod and testicular hormone in the Syrian hamster, Endocrinology 99 (1976) 1528-1533.

[49] M.J. Tetel, M.J. Getzinger, J.D. Blaustein, Fos expression in the rat brain following vaginal-cervical stimulation by mating and manual probing, J. Neuroendocrinol. 5 (1993) 397-404.

[50] F.W. Turek, G.B. Ellis, Steroid-dependent and steroid-independent aspects of the photoperiodic control of seasonal reproductive cycles in male hamsters, in: B.K. Follett, D.E. Follett (Eds.), Biological Clocks in Seasonal Reproductive Cycles, Wright, Bristol, 1981, pp. 251-260

[51] S.R. Wersinger, M.J. Baum, Sexually dimorphic processing of somatosensory and chemosensory inputs to forebrain luteinizing hormone-releasing hormone neurons in mated ferrets, Endocrinology 138 (1997) 1121-1129.

[52] R.I. Wood, S.W. Newman, Mating activates androgen receptor-containing neurons in chemosensory pathways of the male Syrian hamster brain, Brain Res. 614 (1993) 65-77. 